\title{
Physical Parameters of the Detached Eclipsing Binary KIC3858884 with a $\delta$-Scuti Type Pulsating Component
}

\author{
C.-U. Lee ${ }^{1}$, S.-L. Kim ${ }^{1}$, J. W. Lee ${ }^{1}$, K. Pavlovski ${ }^{2,3}$ and J. Southworth ${ }^{3}$ \\ ${ }^{1}$ Korea Astronomy and Space Science Institute, Daejeon 305-348, Korea \\ email: leecu@kasi.re.kr \\ ${ }^{2}$ Department of Physics, University of Zagreb, Bijenička cesta 32, 10000 Zagreb, Croatia \\ ${ }^{3}$ Astrophysics Group, EPSAM, Keele University, Staffordshire, ST5 5BG, UK
}

\begin{abstract}
We present physical parameters for the detached eclipsing binary KIC3858884 which has a $\delta$-Scuti type pulsating secondary component. To derive orbital elements from the radialvelocity curve, high resolution Echelle spectra were obtained at the Bohyunsan Optical Astronomy Observatory in Korea. The BOES spectra and Kepler photometric data were analyzed with well-known codes: JKTEBOP and Wilson-Devinney model for eclipsing light-curve synthesis, and Period04 for pulsation frequency analysis. After the iterative curve fitting, we determined the physical parameters of KIC3858884 as $\mathrm{M}_{1}=2.02 \pm 0.23 \mathrm{M}_{\odot}, \mathrm{M}_{2}=2.02 \pm 0.16 \mathrm{M}_{\odot}, \mathrm{R}_{1}=3.61$ $\pm 0.12 \mathrm{R}_{\odot}, \mathrm{R}_{2}=2.84 \pm 0.10 \mathrm{R}_{\odot}$, respectively.
\end{abstract}

Keywords. stars: fundamental parameters, stars: oscillations, binaries: eclipsing

\section{Introduction}

As the Kepler satellite data archive opened to the public, many interesting light curves with ultra-high precision have been introduced. We compiled interesting targets which show the light variation of a detached eclipsing binary star with a $\delta$-Scuti type pulsation. While Kepler data provide high precision photometry, the data do not contain multi-band photometric information. Therefore, spectroscopic and multi-band photometric follow-up observations are essential to analyze the targets in detail. In this paper, we derive the physical parameters of KIC3858884.

\section{Iterative light curve analysis using PERIOD04 and JKTEBOP}

We used the public data obtained by Kepler in Quarter 2. The photometric data showed clearly short-period sinusoidal variations at out-of-eclipse phases. From the frequency analysis using Period04 (Lenz \& Breger 2005), a total of 198 frequencies which have amplitude signal-to-noise ratio (SNR) of larger than 4 were found at out-of-eclipse phases. Using those frequencies, a synthetic light curve is calculated and then subtracted from the originally observed light curve. The residual light curve was analyzed using JKTEBOP (Southworth et al. 2004). Primary and secondary eclipses are shown on the left side of the Figure 1. The black dots represent Kepler observation data with the eclipsing light variation removed. The green dots represent the synthetic pulsation light curve. In the primary eclipses, the amplitude of black dots is larger than green ones, while the amplitude of black dots is smaller than green ones in secondary eclipses. This is strong evidence that the secondary is a pulsating component; minimizing the dilution effect at the primary eclipse and occultation of a pulsator at the secondary eclipse. 

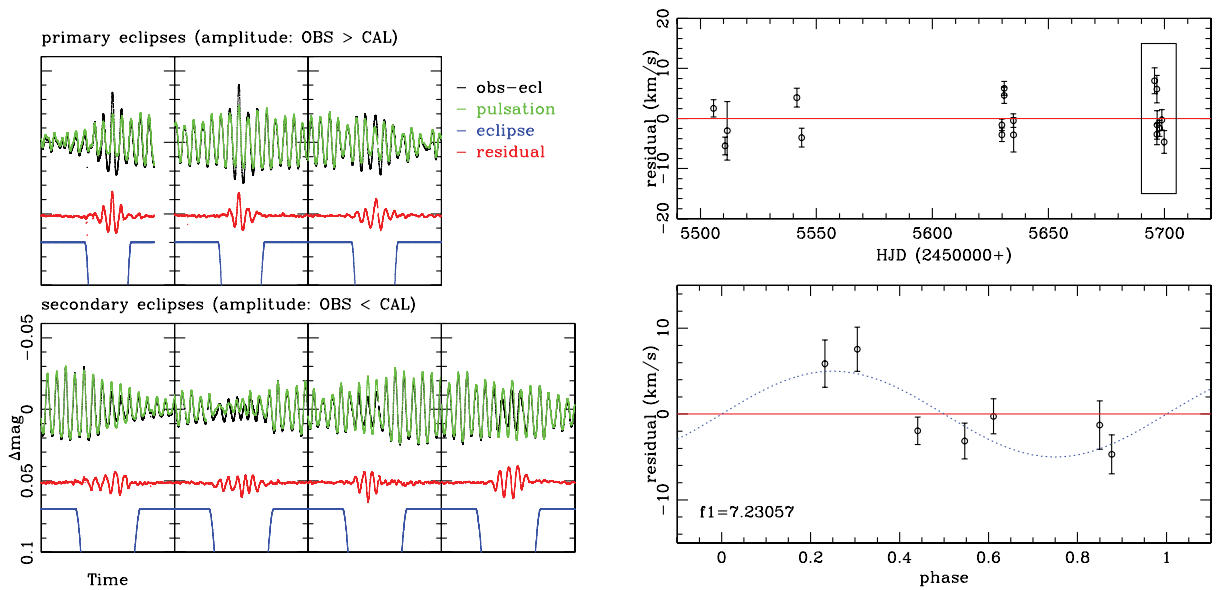

Fig 1. Primary and secondary eclipses are shown with calculated pulsating light curve (left), Residual of secondary RV curve fit and correlation with a dominant pulsation frequency of $\mathrm{f}_{1}=7.23057$ which is almost $3^{h} 20^{m}$ (right)

\section{Radial velocity curve analysis using cross correlation and WD2010}

A total of 18 Echelle spectra were obtained from Nov. 3, 2010 to May 16, 2011 at Bohyunsan Observatory, Korea. The highest SNR of observed spectra is $\sim 200$ and the lowest is $\sim 50$ at near of $6700 \AA$. We used the cross correlation method with a synthetic template spectrum to derive RVs. The optimal parameters (Teff, $\log \mathrm{g},[\mathrm{Fe} / \mathrm{H}]$ and $\xi$ ) were determined by minimizing the difference between the observed and synthetic spectra from the iterative process. Balmer lines and strong lines of the observations were compared to the synthetized spectra. To derive RVs, all observed spectra were cross-correlated with the synthetic spectrum for every $100 \AA$ width from 4000 to $6400 \AA$. The peak of each cross correlation function was measured from the Gaussian fit. The RVs and errors were determined by median filtering of measurements and standard deviation, respectively. WD2010 (Wilson \& Devinney 1971) was used to analyze the RV curve. Scattered residuals were found in the secondary RVs, while no scatter was found in the primary RVs. We suspect this residual is evidence of a pulsating secondary component. We tried to find a relation between the residual of secondary and the dominant pulsation frequency of $f_{1}$. The period of the frequency $\mathrm{f}_{1}=7.23057$ is almost $3^{h} 20^{m}$. The right panel of Figure 1 shows the relation.

\section{Physical parameters of KIC3858884}

From iterative light curve and radial velocity analysis, physical parameters of KIC3858884 were determined. The parameters are $\mathrm{M}_{1}=2.02 \pm 0.23 \mathrm{M}_{\odot}, \mathrm{M}_{2}=2.02 \pm$ $0.16 \mathrm{M}_{\odot}, \mathrm{R}_{1}=3.61 \pm 0.12 \mathrm{R}_{\odot}, \mathrm{R}_{2}=2.84 \pm 0.10 \mathrm{R}_{\odot}$, respectively. In the empirical massradius relation, both components are located above the ZAMS.

\section{References}

Lenz P. \& Breger M. 2005, CoAst, 146, 53

Southworth, J., Maxted, P. F. L., \& Smalley, B. 2004, MNRAS, 349, 547

Wilson, R. E. \& Devinney, E. J. 1971, ApJ, 166, 605 